\title{
RESEARCH
}

Open Access

\section{proBDNF is modified by advanced glycation end products in Alzheimer's disease and causes neuronal apoptosis by inducing p75 neurotrophin receptor processing}

Catherine Fleitas ${ }^{1}$, Gerard Piñol-Ripoll ${ }^{4}$, Pau Marfull1, Daniel Rocandio', Isidro Ferrer ${ }^{2,3}$, Claire Rampon ${ }^{5}$, Joaquim Egea ${ }^{1,6}$ and Carme Espinet ${ }^{6^{*}}$

\begin{abstract}
Alzheimer disease (AD) is a complex pathology related to multiple causes including oxidative stress. Brain-derived neurotrophic factor (BDNF) is a neutrotrophic factor essential for the survival and differentiation of neurons and is considered a key target in the pathophysiology of various neurodegenerative diseases, as for example AD.

Contrarily to BDNF, the precursor form of BDNF (proBDNF) induces apoptosis through the specific interaction with p75 and its co-receptor, Sortilin.

We used hippocampal tissue and cerebrospinal fluid from AD patients and controls. to study the localization and the levels of proBDNF, p75 and Sortilin as well as the post-traduccional modifications of proBDNF induced by Radical Oxygen Species, by immunofluorescence and Western blot. Differentiation and survival were assessed on differentiated mouse hippocampal neurons derived from postnatal neural stem cells from WT animals or from the transgenic AD animal model APP/PS1 $\triangle E$ E, based on mutations of familiar AD. In AD patients we observe a significative increase of proBDNF and Sortilin expression and a significative increase of the ratio proBDNF/BDNF in their cerebrospinal fluid compared to controls. In addition, the proBDNF of AD patients is modified by ROS-derived advanced glycation end products, which prevent the processing of the proBDNF to the mature BDNF, leading to an increase of pathogenicity and a decrease of trophic effects. The cerebrospinal fluid from AD patients, but not from controls, induces apoptosis in differentiated hippocampal neurons mainly by the action of AGE-modified proBDNF present in the cerebrospinal fluid of the patients. This effect is triggered by the activation and processing of p75 that stimulate the internalization of the intracellular domain (ICD) within the nucleus causing apoptosis. Induction of apoptosis and P75 ICD internalization by AD patients-derived proBDNF is further enhanced in neuron cultures from the AD model expressing the APP/PS1 $\triangle E 9$ transgene.

Our results indicate the importance of proBDNF neurotoxic signaling in AD pathology essentially by three mechanisms: i) by an increase of proBDNF stability due to ROS-induced post-traductional modifications; ii) by the increase of expression of the p75 co-receptor, Sortilin and iii) by the increase of the basal levels of p75 processing found in AD.
\end{abstract}

Keywords: Alzheimer's disease, Biomarkers, BDNF, p75, proBDNF, Oxidative stress, Sortilin

\footnotetext{
*Correspondence: carme.espinet@cmb.udl.cat

${ }^{6}$ Serra Húnter fellow, Associate Professor, Generalitat de Catalunya, Barcelona,

Spain

Full list of author information is available at the end of the article
}

(c) The Author(s). 2018 Open Access This article is distributed under the terms of the Creative Commons Attribution 4.0 International License (http://creativecommons.org/licenses/by/4.0/), which permits unrestricted use, distribution, and reproduction in any medium, provided you give appropriate credit to the original author(s) and the source, provide a link to the Creative Commons license, and indicate if changes were made. The Creative Commons Public Domain Dedication waiver (http://creativecommons.org/publicdomain/zero/1.0/) applies to the data made available in this article, unless otherwise stated. 


\section{Background}

Alzheimer's disease (AD) is a neurodegenerative disorder characterized by episodic memory decline at onset and deficits in multiple cortical functions in later stages. Neuropathology hallmarks are senile plaques and neurofibrillary tangles accompanied by deficits in axonal transport, synaptic dysfunction, and neuronal loss. The exact molecular mechanisms that trigger $\mathrm{AD}$ pathogenesis and disease course are not entirely understood. Among them, the increase of oxidative stress related to aging seems to play a central role, not only in $\mathrm{AD}$ but also in other neurodegenerative diseases [1]. There has been much research into the possible involvement of neurotrophins in $\mathrm{AD}$ pathogenesis, including Nerve Growth factor (NGF) and Brain Derived Neurotrophic Factor (BDNF) [2]. This interest was further supported by studies demonstrating the neuroprotective activity against neurodegeneration in different animal models of several neurodegenerative diseases, including $\mathrm{AD}$, which suggested therefore a promising therapeutic role for neurotrophins [3].

BDNF binds to TrkB tyrosine kinase receptor to trigger the neurotrophic signaling including neuron survival, differentiation and synaptic plasticity of various nerve cell populations during normal development and during tissue repair after injury $[4,5]$. BDNF is synthesized as a precursor form (proBDNF) that is processed by the activity of several proteases rendering three main products of $14 \mathrm{kDa}$ (mature BDNF), $28 \mathrm{kDa}$ and $34 \mathrm{kDa}$ [6]. ProBDNF was reported to be biologically active and, unlike BDNF, it was shown to induce apoptosis through its interaction with $\mathrm{p} 75$ and its co-receptor, Sortilin [6-11]. p75 belongs to the family of tumor necrosis factor receptors, has not catalytic activity but a conserved non functional death domain [12]. The signaling mechanisms of p75 are complex and involve several intracellular interactors which trigger specific effects including Sc1, NRAGE and Necdine (cell cycle arrest) [13], RhoA (neuritogenesis) [12] and NRIF (apoptosis) [14]. An important signaling event for p75 involved in apoptosis is the processing of the receptor by convertases, in particular by a $\alpha$-protease (BACE1) followed by a $\gamma$-secretase activity (presenilin 1 or PS1), that yield a $20 \mathrm{kDa}$ intracellular domain (p75ICD) that is translocated into the nucleus [15-18]. p75ICD regulates the activity of the transcription factor NRIF inducing apoptosis [19]. Besides all these functions, p75 has also been reported to act as a co-receptor for Trk, indicating that p75 plays a central role in integrating the neurotrophic effects triggered by neurotrophins (as Trk co-receptor) and the pro-apoptotic signaling triggered by pro-neurotrophins (as Sortilin co-receptor).
In normal brains, the pro-apoptotic function of proneurotrophins and the neurotrophic effects of mature neurotrophins are tightly controlled by their expression levels as well as by the expression levels of their receptors, in particular p75 which is crucial for the integration of the two effects. It is conceivable therefore that neurodegeneration during $\mathrm{AD}$ might be caused by an imbalance of survival and death mechanisms due to changes in the relative levels of these proteins. Accordingly, the cerebral cortex of AD patients showed increased levels of p75 [20, 21] concomitant with decreased levels of Trk receptors which will favor an apoptotic signaling [22-24]. BDNF signaling was also shown to be altered in $\mathrm{AD}$ as an splicing form of $\operatorname{TrKB}$, which gives rise to a truncated form of the receptor lacking the tyrosine kinase domain (presumably acting as a dominant negative), is expressed predominantly in hippocampus and frontal cortex in $\mathrm{AD}$ [23]. On the other hand, several works have described a reduced concentration of BDNF in the CSF of AD-affected patients [24]. Paradoxically, some authors have shown a decrease of proBDNF expression in AD-affected human brains [25, 26]. However, in the CSF of AD-affected patients, the levels of proBDNF have not been analyzed yet. Moreover, there is no data in the literature about the relative levels of proBDNF and BDNF in the CSF of AD-affected individuals compared to controls.

The relative levels of proBDNF and BDNF are directly regulated by the activity of the proteases involved in their processing. We have previously shown that proNGF extracted from brains of AD patients was more resistant to be processed by convertases in comparison to the proNGF isolated from control brains [27, 28]. We reported that the relative stability of proNGF in $\mathrm{AD}$ brains was due to post-traduccional modifications as a consequence of an increase of oxidative stress in the affected tissue [28]. In particular, we observed that Glyoxal (GO) and methylglyoxal (MGO), two highly reactive dicarbonyls that are increased during OS, reacted with free amino groups of Lys, Arg and Cys residues in proNGF, leading to the formation of the AGE/ALE adducts Ne-(carboxymethyl)-lysine (CML), Ne-(carboxyethyl)-lysine (CEL) and intermolecular crosslink and making proNGF in $\mathrm{AD}$ patients more resistant to the action of convertases [28].

In the present work we have studied the relative levels of BDNF and proBDNF and their receptors in AD patients and found a significant increase of Sortilin and proBDNF in the hippocampus. We also detected a significant increase of the ratio proBDNF/BDNF in the CSF of the AD patients, which shows a good correlation with the pathogenic effect of the pro-neurotrophin in the brain. Interestingly, the proBDNF in the CSF of $\mathrm{AD}$ 
patients showed an increase of CEL modifications which account for the increase of the proBDNF/ BDNF ratio due to the major stability of the modified pro-form. Stimulation of primary cultures of hippocampal neurons with CSF from AD patients increased significantly the apoptotic cell death and the nuclear localization of p75ICD compared with the CSF from controls. Similar effects were observed when neurons were stimulated with a proBDNF that was modified in vitro by MGO. Importantly apoptosis and p75ICD nuclear localization were strongly reduced when the CSF of AD patients was immunodepleted of proBDNF, indicating that these effects were mediated, to a large extent, by the presence of proBDNF in the CSF. Finally, we demonstrated that the modified proBDNF had a stronger effect on apoptosis and p75ICD nuclear localization on primary cultures of hippocampal neurons obtained from a transgenic $\mathrm{AD}$ mouse model expressing the APP/PS1 $\triangle \mathrm{E} 9$ transgene. In summary, we propose that in AD, proBDNF-p75/Sortilin signaling has an important contribution to the pathogenesis of the disease, causing an increase of cell death and impairing neuronal differentiation.

\section{Methods}

\section{Human brain samples}

Brain samples were obtained from the Institute of Neuropathology, Bellvitge University Hospital. Brain tissue was obtained from the Institute of Neuropathology HUB-ICO-IDIBELL Biobank and the Hospital ClinicIDIBAPS Biobank following the guidelines of Spanish legislation on this matter (Real Decreto de Biobancos 1716/2011) and approval of the local ethics committees. At autopsy, one hemisphere was rapidly cut in coronal sections $1 \mathrm{~cm}$ thick and selected areas of the encephalon were dissected, frozen on dry ice, and stored at $-80{ }^{\circ} \mathrm{C}$ in labeled plastic bags until use. The other hemisphere was fixed by immersion in $4 \%$ buffered formalin for three weeks for morphologic examination. The neuropathological study was carried out on twenty-five regions of the cerebral cortex, diencephalon, thalamus, brainstem, and cerebellum. De-waxed paraffin sections were stained with hematoxylin and eosin and Klüver-Barrera and processed for immunohistochemistry to microgliaspecific markers, glial fibrillary acidic protein, $\beta$-amyloid, phosphorylated tau, $\alpha$-synuclein, TDP-43, ubiquitin, and p62. Neuropathological diagnosis of the AD was carried out following the Braak, and Braak stages [29] adapted to paraffin sections [30]. Cases with concomitant pathologies, including Lewy body diseases, tauopathies (particularly argyrophilic grain disease), vascular diseases, TDP-43pathies, and metabolic syndrome were excluded. Control and disease cases were processed in parallel. The anterior hippocampus area was used for further immunohistochemical studies.

\section{Human CSF samples}

CSF samples were obtained from control and AD-affected patients and maintained at $4{ }^{\circ} \mathrm{C}$ for less than $4 \mathrm{~h}$. All patients have signed informed consent and the study has been approved by the hospital's ethics committee. All patients underwent lumbar puncture between 8:00 and 10:00 in the morning to avoid variations relating to the circadian rhythm. Protein concentration was determined by DC-Protein Assay (Bio-Rad). Samples were collected and frozen in polypropylene tubes. Post-puncture, CSF was centrifuged at $2000 \mathrm{~g}$ for 10 min at $4{ }^{\circ} \mathrm{C}$ and stored at $-80{ }^{\circ} \mathrm{C}$ until the use of the samples. The levels in CSF A $\beta 42$, t-tau and p-tau were determined by a method of enzyme-immuno assay (ELISA) using the kit Innotest Amyliod $\beta$ (1-42), Innotest htau and phospho-tau 181 (Innogenetics ${ }^{\circ}$ ) according to the manufacturer's instructions. All samples were measured in duplicate and were expressed as $\mathrm{pg} / \mathrm{ml}$. We use cut off points based on the calculation of sensitivity and specificity of our own study population (different from this sample).

\section{Animal model}

APPswe/PS1DE9 with a C57Bl/6 background (double transgenic mice expressing a chimeric mouse/human amyloid precursor protein and a mutant human PS1 with deletion in exon 9) were purchased from The Jackson Laboratory and kept in specific pathogen free conditions under standard animal housing conditions in a 12-h dark-light cycle with free access to food and water in the animal house facility of the Universitat de Lleida [31, 32]. Heterozygous males were bred with wild-type C57/Bl6 females. Animal procedures were conducted according to ethical guidelines (European Communities Council Directive 86/609/EEC) and approved by the local ethics committee of the Universitat de Lleida. For experiments, tail biopsies were taken from $\mathrm{P} 0$ offsprings for genotyping by PCR according to the PCR conditions suggested by The Jackson Laboratory. Mice not expressing the transgene were used as controls.

\section{Cell culture}

Hippocampal Neural Stem Cells (NSCs) were isolated from neonatal pups (P0-P1) from controls and APP/PS1 transgenic mice. Briefly, the brains were dissected in Hanks Medium 1X (Gibco 11,039-021) supplemented with Penicillin-Streptomycin (P/S) (1\%) (Sigma-Aldrich P4333). After dissection, the hippocampi were washed with Hanks Medium with P/S (1\%) and dissociated in Hanks Medium with Papain (Sigma-Aldrich 1,001,992,754) for $20 \mathrm{~min}$ at $37{ }^{\circ} \mathrm{C}$. Papain activity was stopped with two washes with Trypsin inhibitor $(10 \mathrm{mg} / \mathrm{ml}$ in Hanks medium) (Roche Diagnostics 10,109,886,001) plus three washes with $1 \mathrm{ml}$ of Dulbecco's modified Eagle's medium (DMEM)/F12, 
supplemented with B27 (2\%) (Gibco 12,587-010) and P/S. Mechanical dissociation was performed with a heat-rounded Pasteur pipette tip. Dissociated cells were centrifuged at $600 \mathrm{rpm}$ for $4 \mathrm{~min}$, and the pellet was resuspended in DMEM/F12 $\left(37{ }^{\circ} \mathrm{C}\right)$ supplemented with B27 (2\%), P/S(1\%), Epidermal Growth Factor (EGF) $(20 \mu \mathrm{g} / \mathrm{ml})$ (Sigma-Aldrich E9644) and Fibroblast Growth Factor (FGF) $(20 \mu \mathrm{g} / \mathrm{ml})$ (Alomone Labs F-170). Cells were platted on bacterial, non-treated, P35 plates and incubated for four days at $37{ }^{\circ} \mathrm{C}$. After four days, the supernatant was centrifuged at $600 \mathrm{rpm}$ for $4 \mathrm{~min}$, the supernatant was removed and the pellet was washed in complete medium DMEM/ F12 $\left(37{ }^{\circ} \mathrm{C}\right)$ supplemented with B27 $(2 \%)$ and P/S (1\%), without growth factors. Then the neurospheres were dissociated with a pipette in $500 \mu \mathrm{l}$ of complete medium and determined the cell concentration by Trypan Blue dye exclusion. Cells were plated at 6000 cells/well concentration in 8-well on a glass slide coated with poly-D-lysine (Sigma-Aldrich P-7886) and laminin (Invitrogen 23,017015) with complete medium (DMEM/F12, B27 (2\%) and P/S (1\%)). Neurons were treated at 1 DIV. Treatments were done after two washes with DMEM/F12 without B27. The treatments with neurotrophins and controls are done with DMEM/F12 containing 0,25\% B27. For treatments with CSF, all the media is substituted by CSF from controls and $\mathrm{AD}$ with $\mathrm{P} / \mathrm{S}(1 \%)$. After six days (7 DIV in total), were fixed for $30 \mathrm{~min}$ in $4 \%(w / v)$ paraformaldehyde (PFA)/PBS for immunofluorescence assays.

\section{Western blotting}

CSF samples were obtained as described in 2.2., from control and AD-affected patients and maintained at $4{ }^{\circ} \mathrm{C}$ for less than $4 \mathrm{~h}$. Previously to the western blotting, CSF samples were concentrated 20X using Amicon Ultra 10,000 MWCO (Ultracel Low Binding Cellulose) (Millipore). Protein concentration was determined by DC-Protein Assay (Bio-Rad). Proteins were separated by electrophoresis in 12\% SDS-PAGE and transferred to Immobilon-P membranes (Millipore). Membranes were blocked for $1 \mathrm{~h}$ at room temperature in TBS-T $(50 \mathrm{mM}$ Tris, $\mathrm{pH} 8.0$; $133 \mathrm{mM} \mathrm{NaCl}, 0.2 \%$ Tween 20 ) with $5 \%$ skimmed milk and incubated with primary antibodies against the mature BDNF (1:1000 in TBS-T (Alomone) at $4{ }^{\circ} \mathrm{C}$ overnight and with HRP-conjugated secondary antibodies (Jackson) (1:5000 in TBS-T) at room temperature for $1 \mathrm{~h}$. Detection was performed using an ECL chemiluminescence system (Amersham-Pharmacia) following the manufacturer's instructions. For densitometry analysis of the immunoreactive bands, we used ImageJ (http://rsbweb.nih.gov/ij/) with local background subtracted. For each sample, the relative abundance of each BDNF isoform was expressed as a ratio of that particular BDNF isoform related to the total BDNF signal (sum of proBDNF and mature BDNF). The data were shown as the mean \pm standard deviation (SD).
Differences between groups were calculated using a 2-tailed Student's t-test.

\section{AGE modifications of recombinant proBDNF}

Recombinant human proBDNF (Alomone) was modified by the reactive carbonyl specie MGO that react with free amino groups of Lys residues on proteins, leading to the formation of CEL adducts and intermolecular crosslinks [28]. The reactions were performed for $24 \mathrm{~h}$ at $37^{\circ} \mathrm{C}$ by mixing $500 \mu \mathrm{g}$ recombinant protein with $250 \mu \mathrm{l} 50 \mu \mathrm{M}$ GO or MGO and $250 \mu \mathrm{l} 100 \mathrm{mM}$ sodium phosphate. The modified proBDNF was dialyzed using Amicon Ultra-4 Centrifugal Filter Units (Millipore) to remove the excess of MGO and replace the solution with PBS.

\section{Immunohistochemistry}

Microtome sections of fixed and paraffin-embedded human hippocampus $30 \mu \mathrm{m}$ thick were processed free-floating, and collected in slides. Before immunodetection, they were deparaffined, and rehydrated using standard procedures. Epitope retrieval was performed by incubating the slides in $10 \mathrm{mM}$ sodium citrate buffer (pH 6.0) for $20 \mathrm{~min}$ at $95-98{ }^{\circ} \mathrm{C}$ in a water bath. After washing with $\mathrm{PBS}$ at room temperature, sections were permeabilized with PBS with 0, 1\% Triton X-100 (PBST) and blocked for $1 \mathrm{~h}$ at room temperature with 5\% donkey serum in PSBT. Sections were incubated with primary antibodies in blocking solution over night at $4{ }^{\circ} \mathrm{C}$ (rabbit anti-proBDNF, 1:100 (Alomone); mouse anti-CEL, 1:100 (Trans Genic Inc); rabbit anti-ECDp75, 1:100 (Abcam); goat anti-Sortilin, 1:100 (R\&D System)). After washing the sections were incubated for $2 \mathrm{~h}$ at room temperature with the corresponding secondary antibodies in blocking solution containing DAPI 1:2000 (donkey anti-goat Cy3, 1:500; donkey anti-rabbit Alexa488, 1:500; donkey antimouse Cy3, 1:500 (all from Jackson Immunoresearch). Blockage of anti-proBDNF antibody immunoreactivity was performed by incubating the antigenic peptide with the antibody in a proportion 10:1, during $2 \mathrm{~h}$ at room temperature, before the immunohistochemistry procedure. Fluorescence images were imaged on an Olympus Bx51 fluorescence microscope or under a Fluo View FV-1000 Olympus laser-scanning confocal microscope, and micrographs were uniformly adjusted for levels, brightness, and contrast in Adobe Photoshop.

\section{Immunocytochemistry}

Cells were fixed with 4\% PFA in PBS for $30 \mathrm{~min}$ at room temperature, permeabilized with TBST for $60 \mathrm{~min}$ at room temperature and blocked with 5\% donkey serum in TBST. Primary antibodies were diluted in blocking solution and incubated overnight at $4{ }^{\circ} \mathrm{C}$ (rabbit anti-human p75, 1:100 (Promega) to detect intracellular domain, rabbit anti-ECD p75, 1:100 (Abcam) to detect the extracellular domain of 
p75NTR, goat anti-Sortilin, 1:100 (R\&D System); rabbit anti-TrkB, 1:100 (H-181, Santa Cruz Biotech.); goat anti-SV2,1:100 (DSHB); mouse anti-ßIII tubulin, 1:100 (Sigma); mouse anti-doublecortin, 1:100 (Cell Signaling), rabbit anti-calbindin, rabbit anti-calretinin and parvalbumin,1:100 (Swant)). After washing samples were incubated for $2 \mathrm{~h}$ at room temperature with the corresponding secondary antibodies diluted in blocking solution containing DAPI 1:2000 (monkey anti-goat Cy3, 1:500; donkey anti-rabbit Alexa488, 1:500; donkey anti-mouse Cy3, 1:500 (all from Jackson Immunoresearch)). Fluorescence images were acquired on a confocal microscopy setup (Olympus FV1000, 60X PlanApo using software Fluoview v.4.3) or on an inverted fluorescence microscope (OlimpusIX71, 20X LCPlanFl).

\section{Quantitative imaging}

All microscope settings were set to collect images below saturation and were kept constant for all images taken in one experiment. Statistical significance ( $p$ values) was assessed using the two-tailed Student's t-test unless otherwise indicated. Results were shown as the mean $+/-$ standard deviation (SD).

\section{Statistical analysis}

Statistical analysis of data was performed using SPSS statistical software (SPSS for Windows, v.16, SPSS, Inc., Chicago, IL). Student's t-test and One-way ANOVA analysis are used to identify significant differences.

\section{Results}

\section{proBDNF expression and the ratio Sortilin/p75 are} increased in the hippocampus of AD patients

The hippocampus is one of the brain regions most affected in the AD $[29,30]$. Particularly vulnerable are a group of neurons located in the hilus, also known as hilear Mossy cells [33, 34]. We obtained brain samples containing this region from $\mathrm{AD}$ patients and controls as summarized in Table 1 and using antibodies directed

Table 1 Summary of the patients from whom hippocampal samples were obtained and studied

\begin{tabular}{llllll}
\hline N & Age & Gender & Diagnostic & Braak Stages & Post-mortem delay /hours \\
\hline 1 & 46 & F & C & 0 & 9 \\
2 & 47 & M & C & 0 & 5 \\
3 & 24 & F & C & 0 & 6 \\
4 & 79 & M & AD & V/B & 5 \\
5 & 82 & F & AD & V/B & 2 \\
6 & 79 & M & AD & V/C & 7 \\
7 & 85 & F & AD & VI/C & 12
\end{tabular}

$C$ control, $A D$ Alzheimer's Disease, $A, B$ and $C$ Braak and Braak's classification of AD stages depending on amyloid plaques, $0-V$ Braak and Braak's classification of $A D$ stages depending on the distribution and amount of neurofibrillary tangles against the extracellular domain of p75 (p75ECD), Sortilin and the pro-domain of BDNF, we performed immunofluorescence assays to determine the levels of these proteins in the two groups. We observed that fluorescence intensity for p75 in individual hileal cells of $\mathrm{AD}$ brains was not significantly different on average compared to control brains (Fig. 1b, f, i). However, Sortilin fluorescence as well as the percentage of double positive cells for Sortilin and p75 increased significantly in AD brains (Fig. 1c, g, i). We also observed an abundant expression of proBDNF in the cytoplasm of the hileal neurons of human hippocampus (Fig. 1k, m).

However, in $\mathrm{AD}$ brain samples, fluorescence intensity of proBDNF was significantly higher than in control brains (Fig. 1k, m, n). Next we asked whether proBDNF and BDNF could be detected in the CSF of AD patients and controls since this technique has been traditionally used to study the expression profile of different markers in alive individuals for the diagnostic and/or the prognostic of neurodegenerative diseases. In this case, we analyzed the CSF by western blot and used antibodies against the mature form of BDNF that also detects the proBDNF. Levels of mature BDNF (14 kDa band) in CSF were very low compared to proBDNF ( $34 \mathrm{kDa}$ band) and required longer exposure times that were shown in a separate panel (Fig. 1o). Densitometric analysis of the two bands in $15 \mathrm{AD}$-affected patients and 15 control individuals (see Table 2 summarizing the demographic and clinical characteristics of CSF donors) revealed that the total levels of BDNF (proBDNF+BDNF) were similar between the two groups (Fig. 1p). However, in AD patients we observed an increase of proBDNF associated with a decrease of BDNF, compared to controls (Fig. 1o). When we calculated the ratio proBDNF/BDNF in each sample, we observed that the average ratio proBDNF/ BDNF was significantly higher in the CSF from AD patients (Fig. 1p). These results indicate that the CSF is a reliable sample that recapitulates the changes in proBDNF/BDNF expression that take place in the brain of AD patients. In summary, we conclude that hileal neurons of $\mathrm{AD}$ patients are more susceptible to cell death due to the higher levels of proBDNF and Sortilin expression and the higher proportion of cells expressing both p75 and Sortilin.

\section{AGE modifications in proBDNF are increased in the hippocampus and the CSF of AD patients}

The increase of oxidative stress in the brain with aging has been proposed to be a key factor triggering and/or enhancing $\mathrm{AD}[1,34]$. The molecular mechanisms involved are diverse and include post-traductional modifications of specific proteins. For instance, GO and MGO, two highly reactive dicarbonyls that are increased during OS, react with free amino groups of Lys, Arg and Cys 


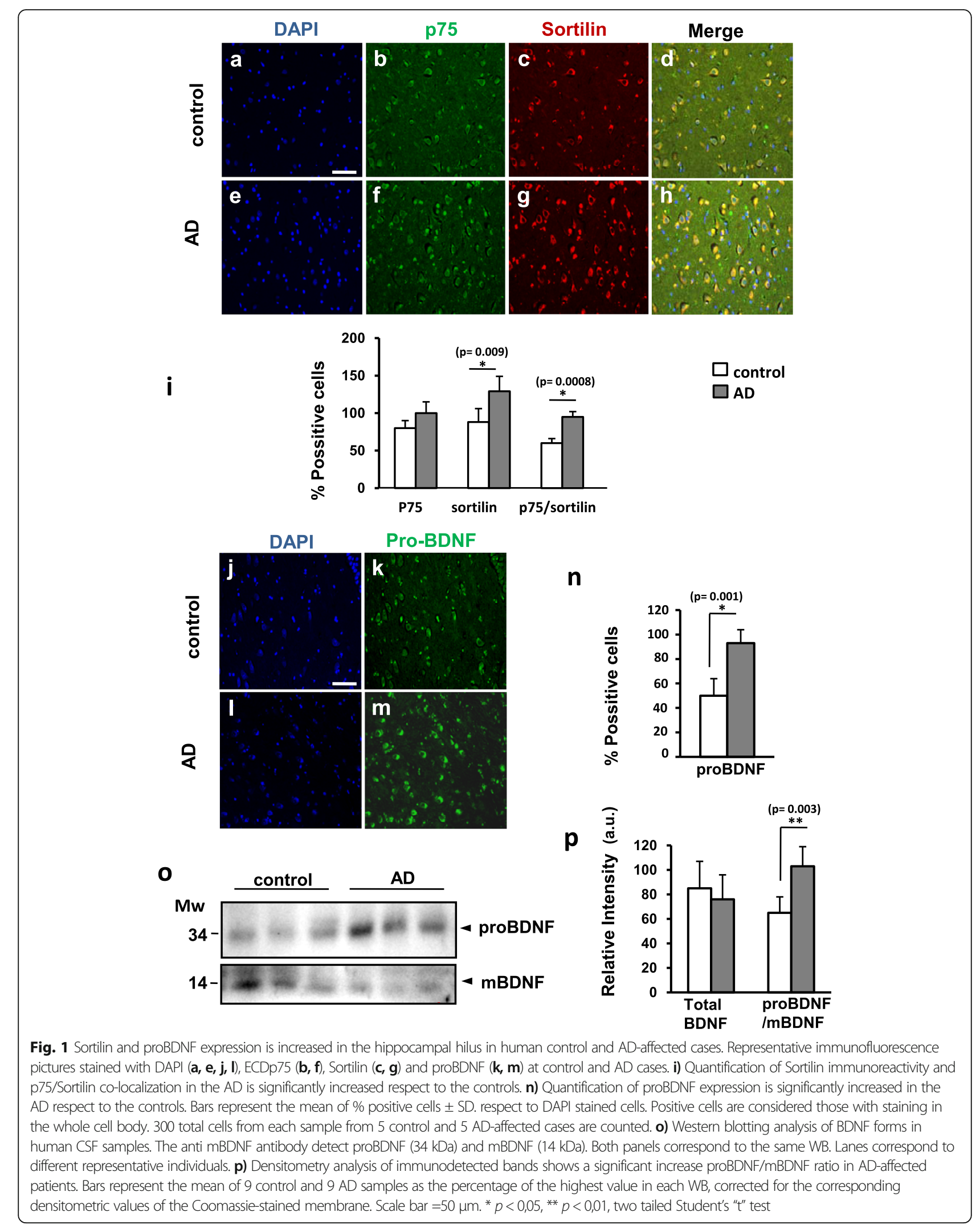


Table 2 Demographic and clinical characteristics of the patients from whom CSF samples were studied. Case (AD); control (C); $p$ (Student's t-test)

\begin{tabular}{llll}
\hline & Case $(n=15)$ & $C(n=15)$ & $P$ \\
\hline Male & $4(26.7 \%)$ & $5(33.3 \%)$ & 0.7 \\
Years & $73.5 \pm 12,1$ & $70.5 \pm 7,1$ & 0.6 \\
Age schooling & $11.3 \pm 5,0$ & $11.3 \pm 2,2$ & 0.2 \\
MMSE & $19,1 \pm 6,0$ & $28,1 \pm 1,8$ & 0 \\
Family history & & & \\
Presenilin AD & $2(13.3 \%)$ & $0(0 \%)$ & 0.14 \\
EA > 65 years & $3(20.0 \%)$ & $5(33.3 \%)$ & 0.4 \\
Pathological history & & & \\
Hypertension & $6(40 \%)$ & $8(53 \%)$ & 0.46 \\
Diabetes & $3(20.0 \%)$ & $2(13.3 \%)$ & 0.6 \\
Hypercolesterolemia & $2(13.3 \%)$ & $7(46.7 \%)$ & 0.04 \\
Depression & $2(13.3 \%)$ & $7(46.7 \%)$ & 0.04 \\
CSF AD Biomarkers & $\mathbf{p g} / \mathbf{m l}$ & $\mathbf{p g} / \mathbf{m l}$ & \\
Amyloid $\beta$ & 386,128 & $856 \pm 204$ & 0 \\
Total Tau & $615 \pm 270$ & $281 \pm 103$ & 0.01 \\
PhosphoTau & $85 \pm 28$ & $55 \pm 18$ & 0 \\
\hline
\end{tabular}

residues leading to the formation of the AGE/ALE adducts CML, CEL and intermolecular crosslink $[35,36]$. As we have previously reported, these modifications affect proNGF during $\mathrm{AD}$ making the pro-neurotrophin form of NGF more stable (as it cannot be converted to mature NGF), which in turn increases cell death [28]. To determine whether proBDNF could be also modified by reactive dicarbonyls in $\mathrm{AD}$, we performed different approaches. First, we studied the co-localization of proBDNF and CEL by immunohistochemistry in the hileal region of human hippocampal samples from controls and AD-affected brains. We observed a remarkable increase of CEL immunoreactivity in AD brains compared to controls (Fig. 2c, g). Interestingly, almost all the cells in the hillial region of AD patients with significant CEL staining, also co-expressed proBDNF, while in control brains very few neurons displayed double immunoreactivity (Fig. 2b-d, f-h, i). These results strongly suggested that proBDNF could be modified by reactive dicarbonyls. In order to address this question directly we performed a more specific assay. We immunoprecipitated proBDNF from the CSF of controls and AD patients and analyzed the precipitates by Western blot with an antibody against CEL. We found that the proBDNF in the CSF from AD patients showed a prominent CEL modification, around 6-fold on average, compared to controls (Fig. 2j, k). Altogether these results indicate that the proBDNF in AD patients displays AGE post-traduccional modifications as consequence of an increase of oxidative stress during neurodegeneration that might prevent the action of convertases to produce mature BDNF (see below).

\section{proBDNF modified in vitro by MGO induces neuronal apoptosis and decreases neuron differentiation}

In order to test the effects of the AGE modifications of proBDNF induced by oxidative stress conditions on neuron survival and differentiation, we treated recombinant proBDNF in vitro with reactive oxygen species that react with Arg and Cys residues, resulting in the formation of intermolecular modifications and crossovers of the protein (see Methods section). This modified proBDNF (MproBDNF) was used to stimulate primary cultures of neurons along with mature BDNF, unmodified proBDNF as well as an unrelated protein, Bovine Serum Albumin (BSA), modified in the same way as the proBDNF (MBSA), as stimulation controls. In these experiments we used differentiated hippocampal neurons obtained from hippocampal NSCs plated on laminin-coated plates (see Methods). At 1 day in vitro (DIV), the neurons started to differentiate but it was between 4 and 7 DIV that the degree of maturation is significative. In longer culture periods, neurons displayed a complex degree of neuritogenesis and differentiation, mainly in the mBDNF treated wells, which was difficult to quantify. Differentiated neurons expressed doublecortin (DCX), BIItubulin, SV2, p75, Sortilin, TrkB and calretinin (Fig. 3, Additional file 1: Figures S1-S3). These neurons, however, were negative for parvoalbumin and calbindin (Additional file 1: Figure S3), indicating that they are young granular differentiated neurons. In control cultures treated with mature BDNF for 6 DIV, neurons increased significantly their differentiation compared to unstimulated cultures, as demonstrated by $\beta$ IIItubulin staining (Fig. 3a). Interestingly, control stimulation with proBDNF, without AGE modifications, did not increase apoptotic cell death over basal levels; in contrast, it produced similar effects on neuron differentiation as the mature BDNF (Fig. 3a, b). However, stimulation with MproBDNF, produced a significant increase of apoptosis and impaired neuron differentiation (Fig. 3a, b, c). These effects were specific for the AGE modified form of proBDNF (MproBDNF) since they were not observed upon stimulation with MGO modified BSA (MBSA) (Fig. 3a, b, c). These results suggest that proBDNF is quickly converted into the mature form in culture, inducing nearly the same effects as the BDNF, while the MproBDNF, which is more resistant to the action of convertases, is more stable and triggers the adverse effects associated with its pro-apoptotic activity.

\section{CSF from AD patients induces neuronal apoptosis through proBDNF/p75}

Our results have shown so far that in $\mathrm{AD}$ patients there is an increase of proBDNF/BDNF expression ratio as well as 

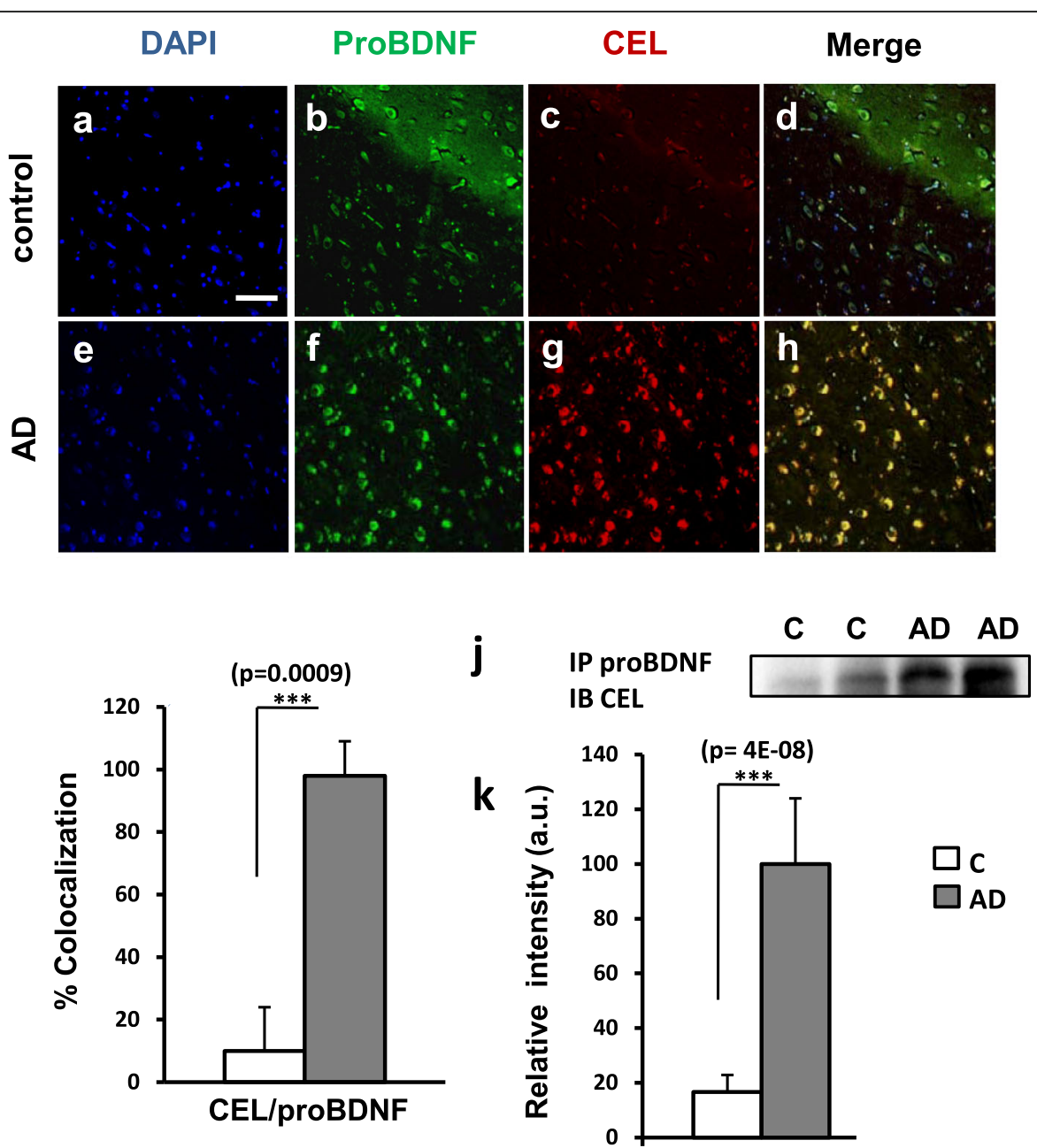

Fig. 2 Hippocampal proBDNF is modiffied by AGE in AD human samples. Representative immunofluorescence pictures stained with DAPI $(\mathbf{a}, \mathbf{e})$, anti-proBDNF $(\mathbf{b}, \mathbf{f})$, anti-CEL $(\mathbf{c}, \mathbf{g})$ at control and AD cases. i) Quantification of CEL and proBDNF co-localization in the AD is significantly increased respect to the controls. Positive cells are considered those with staining in the whole cell body. Bars represent the mean of 5 control and $5 \mathrm{AD}$ samples as \% colocalization of CEL and proBDNF respect to the total cell number (DAPI staining) \pm SD . 300 total cells from each sample are counted. $\mathbf{j}$ ) Representative image of Immunoprecipitation of proBDNF from CSF from controls and AD patients analyzed by Western-blot with antibodies against CEL. k) Bars represent the mean of 9 control and 9 AD samples as the percentage of the highest value in each WB, corrected for the corresponding densitometric values of the Coomassie-stained membrane.Scale bar $=50 \mu \mathrm{m} .{ }^{* * *} p<0,001$, two tailed Student's "t" test

an increase of expression of some of the key signaling elements involved in the pro-apoptotic effects of this pro-neurotrophin. Moreover, we have observed that proBDNF from AD patients displays AGE post-traductional modifications as a consequence of the increase of the oxidative stress during neurodegeneration. Finally, our in vitro experiments on differentiated neurons suggest that these modifications produce a more stable form of proBDNF, a process which can account for 1) the increased levels of proBDNF in AD brains and CSF and 2) the contribution of proBDNF to the cell death associated with the disease. To evaluate further this hypothesis we directly evaluate the effects of the proBDNF contained in the CSF of AD patients and controls on differentiated primary neurons, as above. Thus, we stimulated the cultures for 6 DIV with CSF (substituting all the culture media, see Methods) from control and AD patients and stained the neurons with antibodies against p75ICD or with DAPI staining, in order to assess the subcellular localization of the protein by confocal microscopy and the apoptotic cell death, respectively (Fig. 4a and Additional file 1: Figure S3). Control CSF-treated neurons survived and differentiated normally showing a basal rate of apoptotic cell death of around 10\% (Fig. 4a and c). A high proportion of these neurons displayed a peripheral distribution of p75 in the soma consistent with the presence of the intact receptor in the plasma membrane (Fig. 4a, c). In contrast, cultures treated with ADCSF showed an altered 


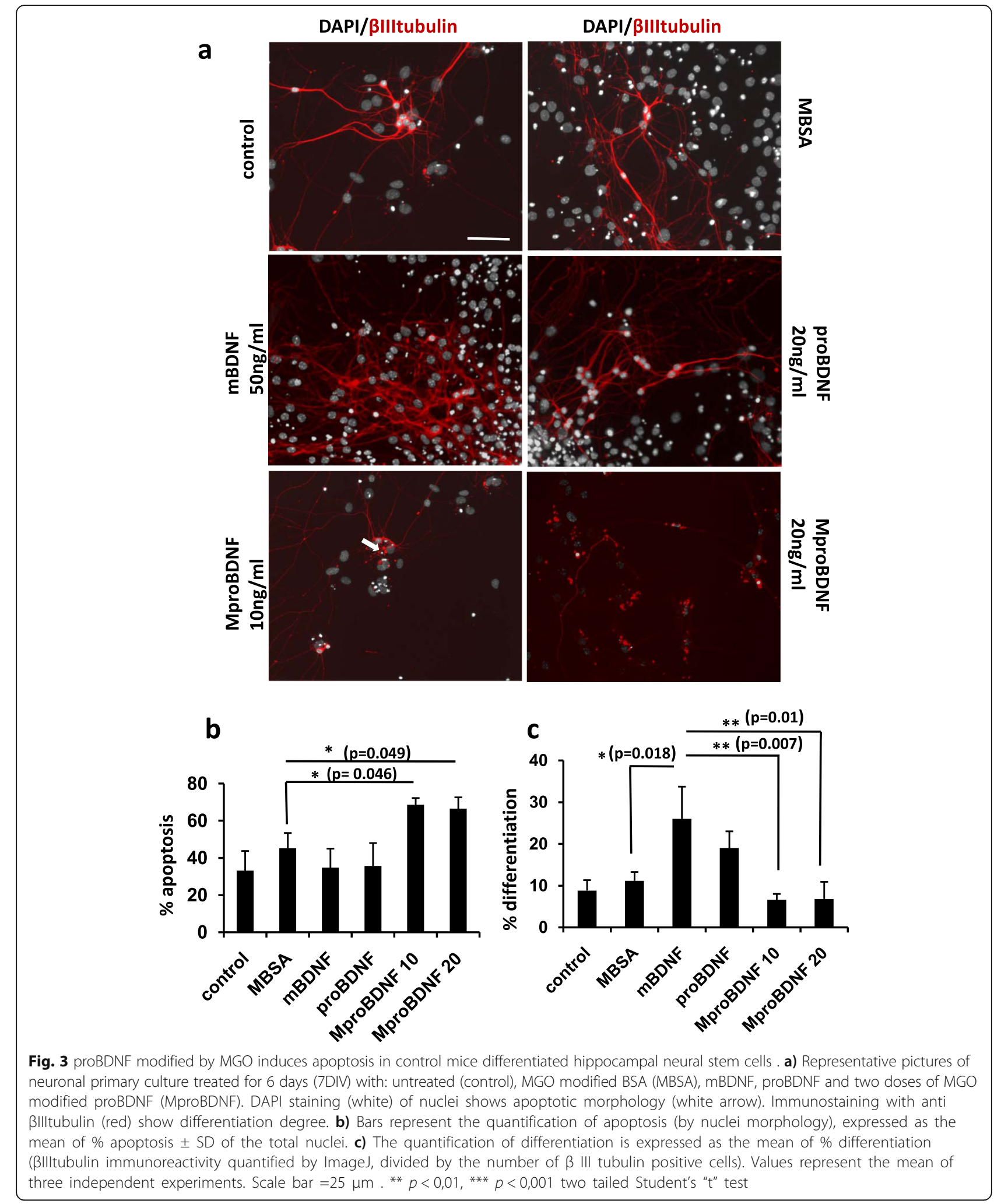

morphology with impaired differentiation and a dramatic increase the number of apoptotic nuclei (reaching 60\%) (Fig. 4a, c). Moreover, under ADCSF treatment, there was a significant increase in the percentage of neurons with nuclear immunoreacitvity for $\mathrm{p} 75$, indicating that the $20 \mathrm{kDa}$ ICD fragment was shed and translocated to the 


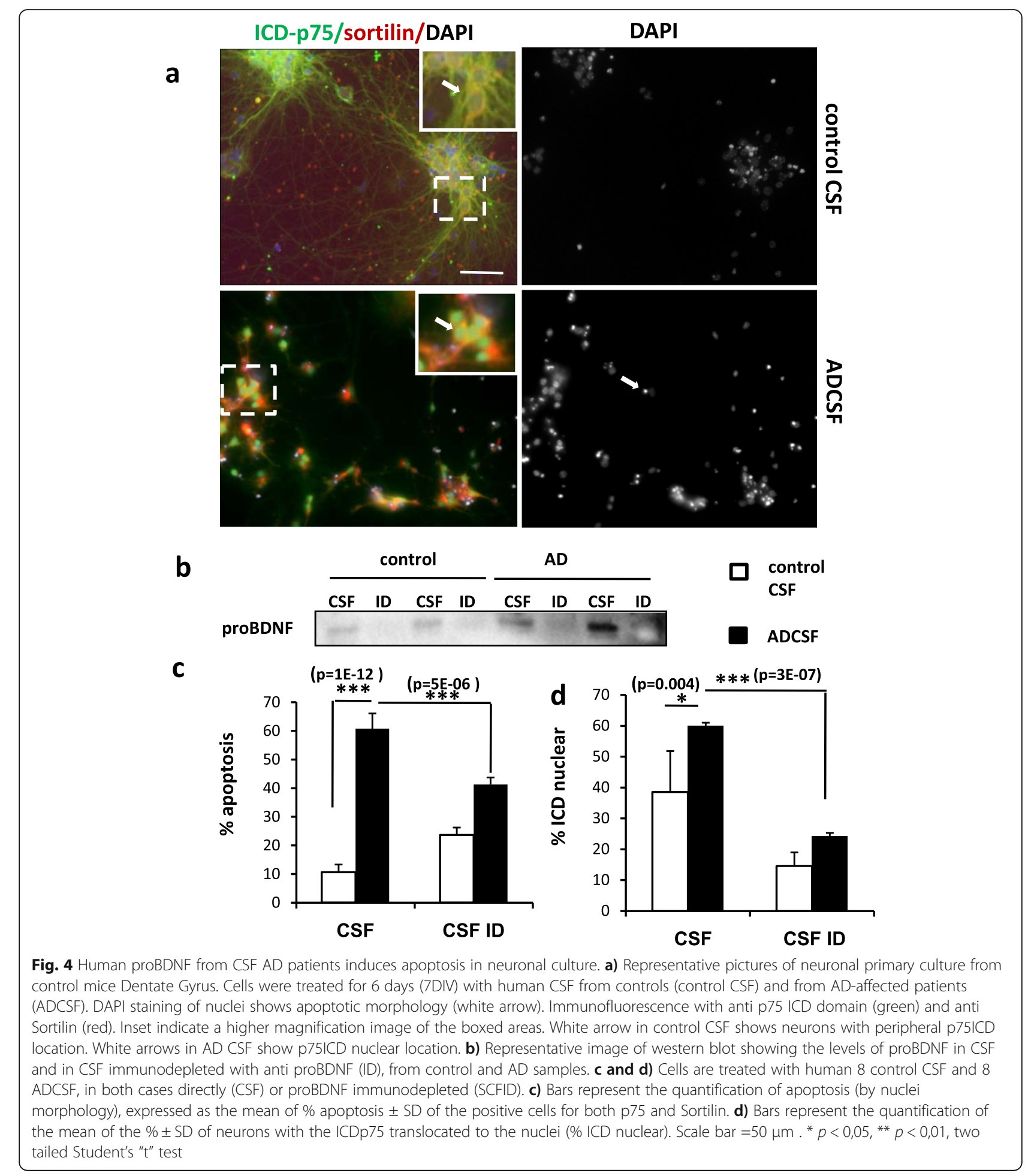

nucleus (Fig. 4a, d). The CSF from AD patients is known to contain several other factors, such as $\mathrm{Au}$, that might be responsible for these neurotoxic effects. To determine whether proBDNF present in the CSF of AD patients we specifically immunodepleted the CSF samples from proBDNF with specific anti-proBDNF antibodies coupled to sepharose AG beads. Successful depletion was demonstrated by Western blot with antibodies against proBDNF (Fig. 4b). We then compared the effects of the immunodepleted CSF (CSFID) and the normal CSF from both, AD patients and controls, in our neuron cultures after 6 DIV. As shown in Fig. 4c and d, depletion of proBDNF 
produced a remarkable reduction of the percentage of apoptosis as well as the percentage of neurons with nuclear immunoreactivity for p75. Therefore, these results indicate that the proBDNF in the CSF of AD patients is biologically active and that it is the main responsible of the pro-apoptotic effects triggered by AD CSF. Moreover, these results strongly suggest that the effects of proBDNF in AD CSF are mediated by activation of p75 signaling.

\section{APP/PS1 mutations increase p75 processing and apoptosis induction}

In the familiar $\mathrm{AD}$, one of the most frequent targets of inherited mutations is the presenilin-gamma secretase complex [37]. Some of these mutations affect mainly presenilin 1 (PS1) including the deletion of exon 9 (u9) or M146 V [38]. These are supposed to be gain of function mutations leading to an increase of protease activity [39]. The study of the pathogenic mechanisms underlying these mutations and the development of $\mathrm{AD}$ has been focused on several of the PS1 substrates, mainly A $\beta$ [39]. Since p75 is also a substrate of PS1 activity [18, 40], we hypothesized that the harmful consequences of proBDNF on neuron survival and differentiation in $\mathrm{AD}$, due to its higher stability as a consequence of AGE modifications, could be boosted by the effects of PS1 mutations on p75 signaling. In particular, we were interested in ascertain whether the shedding of p75, which requires PS1 activity, changes during AD and whether these changes can sensitize the effects of pro-neurotrophins. To test this hypothesis we set up primary neuron cultures from NSCs from heterozygous APP/ PS1 mice or from wild type littermates as controls (see Methods). This APP/PS1 animal model has been widely used in studies of neurological disorders of the brain, specifically $\mathrm{AD}$, amyloid plaque formation, and aging [41]. After 6 DIV, when all the conditions are considered for the analysis (regardless to the treatment), APP/PS1 cultures does not show a \% of apoptosis significantly different than WT (One-way ANOVA $p=0.1$ ). However under treatment with MBSA (control), these transgenic APP/PS1 neurons displayed higher percentage of apoptotic nuclei and higher percentage of neurons with nuclear immunoreactivity for p75 (Fig. 5a, b, c and Additional file 1: Figure S3). Interestingly, stimulation with MproBDNF induced a stronger and significant increase in cell death and p75 nuclear immunoreactivity in these transgenic neurons compared to controls (Fig. 5a, b, c). We therefore conclude that PS1 mutations involved in familiar $\mathrm{AD}$ potentiate the effects of proBDNF on cell death most probably by increasing p75 signaling and, in particular, p75ICD nuclear translocation.

\section{Discussion}

The pathogenic mechanisms underlying the development of $\mathrm{AD}$ are complex and yet not fully understood.
Some important research lines have focused on the role of neurotrophins in $\mathrm{AD}$ both, as regulating factors of cell survival and cell death, involved in the pathogenesis and/or the course of the disease, and as therapeutic tools $[2,3]$. Today, most of our knowledge about the role of neurotrophins in $\mathrm{AD}$ comes from the study of the NGF and its precursor form, proNGF. Thus, proNGF has been reported to be increased during aging and $\mathrm{AD}$ in vulnerable regions such as the hippocampus [42]. Moreover, the proNGF isolated from $\mathrm{AD}$ patients displays higher neurotoxicity than proNGF from controls [27]. More controversial is the role of proBDNF/BDNF alterations in the pathogenicity of the $\mathrm{AD}$. In part, because contradictory evidences were reported regarding the expression and secretion of BDNF in its precursor form $[6,43,44]$. It was suggested that these opposing results reflect a more complex regulation of proBDNF processing that depends on the regulation by third-parties such as glial cells and plasmin inhibitors [45]. Indeed, BDNF is more important for the maintenance and function of central nervous system than NGF and thus, reduced levels of BDNF, due to impairment of proBDNF processing, could be involved in some aspects of the AD disease, such as depression, in addition to the neurotoxic effects of the pro-neurotrophin [46].

In the present work we have addressed the role of proBDNF and its pro-apoptotic signaling mechanisms in $\mathrm{AD}$. We showed that in brains from AD patients there is an increase of the levels of proBDNF are increased, as are also some of the key signaling components involved in its pro-apoptotic effects, such as the co-receptor Sortilin. We were also able to detect an increase of proBDNF in the CSF of AD patients. Interestingly, this increase of proBDNF in the CSF is associated with a decrease of BDNF. Moreover, we have demonstrated that the proBDNF in the CSF from AD patients is highly modified by oxidative stress and that the presence of this AGE modified proBDNF induces apoptosis and impairs differentiation on primary cultures of neurons. Finally, we have shown that the damaging effects of proBDNF can be potentiated by mutations commonly found in familiar forms of AD that affects PS1 protease activity. This synergistic effect is due to the enhancement of p75 signaling, in particular of p75ICD internalization. The present results further reveal the important role of neurotrophin signaling in the development of the AD and, in particular, our results provide evidence for the relevance of the proBDNF/p75 pathway in the pathogenesis and/or the course of AD. Thus, these results highlight the importance of proBDNF/p75 signaling as potential targets to develop novel pharmacological therapies to fight AD.

During aging and especially during neurodegeneration there is an increase of oxidative stress in the tissue that 


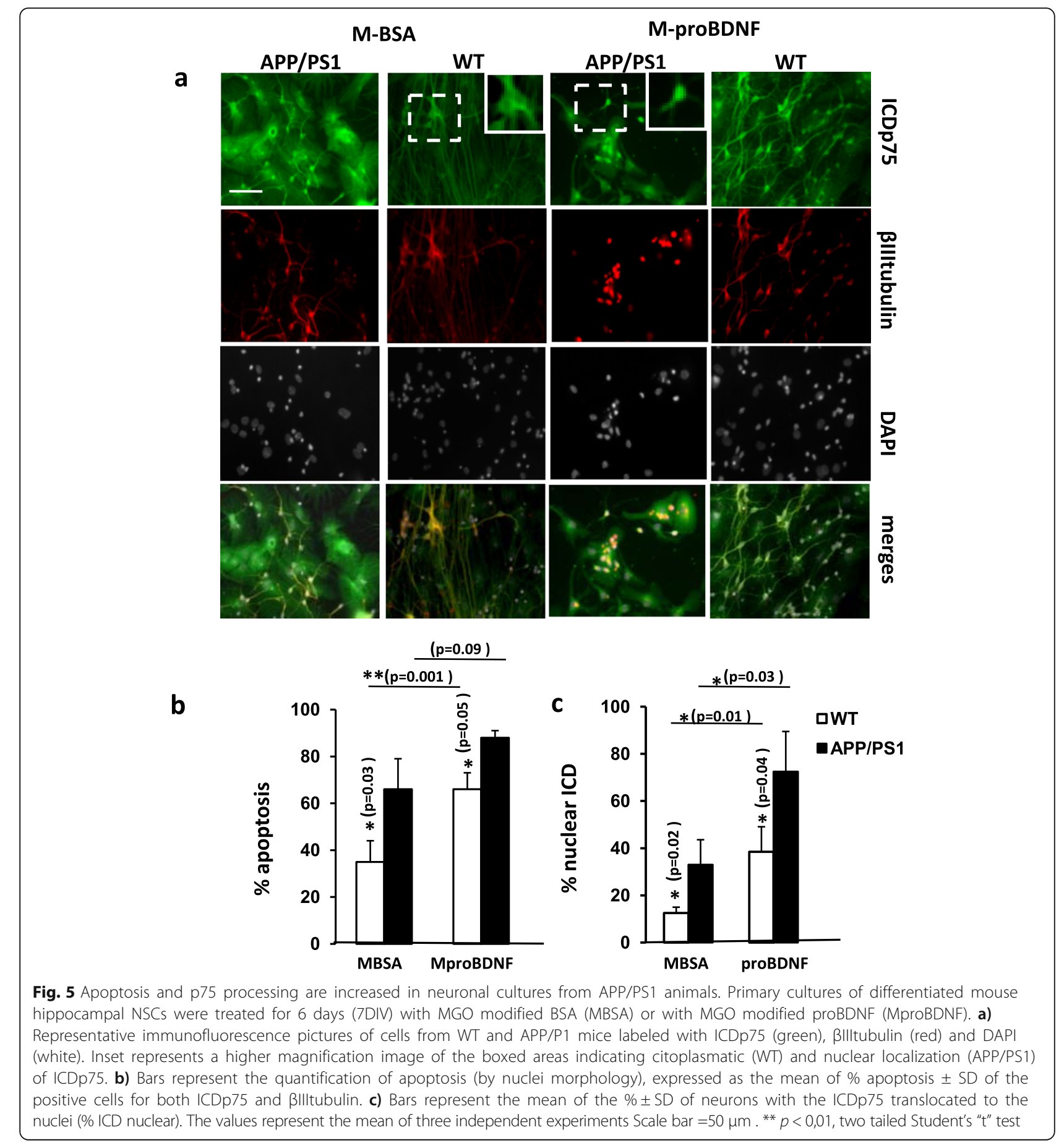

contributes to the pathogenesis of AD [1]. The neurotoxic mechanisms involved, however, are not clearly understood. Glyoxal (GO) and methylglyoxal (MG) are highly reactive dicarbonyl formed during the metabolism that, in excess, can increase ROS production and cause oxidative stress. $\mathrm{GO}$, and MGO can react with free amino groups of Lys, Arg and Cys residues, leading to the formation of the AGE/ ALE adducts CML, CEL and intermolecular crosslink [47]. The relationship between protein carbonylation and neurodegeneration (ND) has been widely established. Changes in protein carbonyl samples from both disease experimental animal models and patients with neurodegeneration have been reported [48, 49]. Moreover, mass spectrometry analysis of human brain homogenates has shown that several AGE/ALE adducts, HNEL, CEL, CML and $\mathrm{N} \varepsilon$-(malondialdehyde)-lysine (MDAL) increase in ND $[1,35]$. Oxidative non-enzymatic modifications can also affect protein structure and function by other means, for 
example increasing protein crosslinking and thus contributes to protein aggregation during ND [1]. These general effects on protein structure might affect the function of some proteins in a specific manner. In the present work, we found that proBDNF co-localizes almost entirely with CEL in AD samples and that the proBDNF isolated from the CSF of AD patients display higher CEL modifications than controls. We therefore suggest that the increase of proBDNF in AD patients, in brain samples and in CSF, is due to a higher stability of this precursor form resulting from post-traductional modifications triggered by oxidative stress that make this modified pro-neurotrophin more resistant to be processed by convertases into the mature BDNF. The target sequence for Furin and other convertases (plasmin and metalloproteases) contains Lys, Arg, and Cys residues that are indeed suitable sites for AGE/ALEs formation [50]. Similar results were obtained by our group when we analyzed the role of proNGF/NGF in human hippocampal samples from $A D$ patients [16, 27, 28]. We cannot rule out the possibility that AGE modified proBDNF could have acquired gain-of-function effects independent of proBDNF signaling. Nevertheless, since there is a strong correlation between proBDNF levels in $\mathrm{AD}$ and p75 signaling (see below) we rather favor the hypothesis these oxidative stress-induced modifications makes the proBDNF more stable and tip the balance in favour of cell death (increase of proBDNF) at expenses of cell survival (decrease of BDNF). Accordingly, we observed in the CSF of AD patients an increase of proBDNF that was associated with a decrease of BDNF. In a more functional approach we demonstrated that the CSF from AD patients, but not the CSF from controls, induces apoptosis in a primary culture of differentiated neurons obtained from NSCs from the Dentate Gyrus. In contrast, CSF from control individuals maintained the survival and differentiation of these neurons up to $6 \mathrm{DIV}$. Interestingly, depletion of proBDNF from AD CSF samples reduced strongly the apoptotic effect of the original AD CSF (apoptosis reduced from 40 to 20\%), suggesting that proBDNF is a main factor that triggers the apoptotic effect of the AD CSF. The remaining 20\% apoptosis could be due to other pro-apoptotic inducers such as $A \beta$ or proNGF (references). Finally, we propose that the ratio proBDNF/BDNF measured in the in the CSF could be a potential diagnostic marker for $\mathrm{AD}$. To further validate this approach, it would be interesting to measure the proBDNF/BDNF ratio in the CSF of a larger cohort of patients and controls and analyze this ratio in regard to the different stages of the disease.

BDNF genetic variation may affect the risk of developing $\mathrm{AD}$ [51]. One of these variations, proBDNF ${ }^{\text {Val66Met, }}$ impairs the normal processing of proBDNF resulting in higher levels of expression of proBDNF [52]. The Val66Met polymorphism affects a particular step of proBDNF processing that is sufficient to impair the formation of enough levels of mature BDNF and trigger familiar depression [46]. The mutation blocks proBDNF maturation and affects activity-dependent secretion of BDNF and human memory and hippocampal function [53, 54] and worsens vulnerability to stress and response to antidepressants [55]. Despite the proBDNF ${ }^{\mathrm{Val} 66 \mathrm{Me}}$ is able to interact with p75 and Sortilin, altering the neuronal morphology [52], a recent meta-analysis did not show an association of the Val66Met polymorphism and the risk of $\mathrm{AD}$ [56]. It is possible that the proBDNF ${ }^{\mathrm{Val} 66 \mathrm{Met}}$ is still partially processed reducing the neurotoxic effects of the full proBDNF form.

Besides the increase of proBDNF levels in AD patients, we provide evidence for the existence of at least two additional mechanisms that could potentiate the neurotoxic action of proBDNF and accelerate the disease. First, we observed that the levels of Sortilin, the co-receptor of p75NTR involved in the pro-apoptotic effect of proBDNF, are increased in the hileal region of the hippocampus of $\mathrm{AD}$ patients thus making the neurons of this region more vulnerable to cell death. Sortilin levels seem to be particularly sensitive to ageing and neurodegeneration. For instance, Sortilin immunoreactivity was described to be increased in ageing rodent basal forebrain and sympathetic neurons while the levels of p75NTR were either unchanged or reduced [57]. On the other hand and similarly to our results, Sortilin was shown to be increased in the temporal cortical area of human AD brains as well as in brains of 6-months old PS1delta9 transgenic mice [58]. Interestingly, this increase was suggested to be triggered by amyloid beta $_{1-42}\left(A \beta_{42}\right)$ through the p75/RhoA signaling pathway suggesting a potential physiological interaction of $A \beta_{42}, p 75 N T R$ and sortilin in AD [57]. From all this data we propose that neurodegeneration in $\mathrm{AD}$ patients may be enhanced by an increase of sortilin expression in neurons, probably induced by $A \beta_{42}$, together with elevated levels of proBDNF in specific regions.

Secondly, we found that proBDNF effects in AD can also be enhanced by mutations found in familiar AD that affect the convertase activity of the $\gamma$-secretase PS1. We propose that these mutations make neurons more sensitive to the pro-apoptotic effect of proBDNF by increasing the susceptibility of p75 to be processed and induce nuclear translocation of the p75ICD. Although in familiar AD PS1 mutations are described to increase Aß42/40 ratio, the effect of these mutations on its convertase activity as gain or loss of function is controversial [39]. In the present work, we have observed an increase of basal cleavage of p75 and an increase of p75ICD nuclear translocation and apoptosis (further induced by MproBDNF), in neurons from the animal model of AD APP/PS1 bearing the mutation $\Delta 9$ in PS1. Therefore we favor the idea that, 
at least the $\Delta 9$ deletion in PS1, behaves as a gain of function mutation.

More than fifty membrane proteins, including APP and $\mathrm{p} 75$, are cleaved by the presenilin1- $\gamma$-secretase complex $[59,60]$. APP processing and $A \beta$ production is one of the hallmarks of $\mathrm{AD}$ [29]. On the other hand, the cleavage of p75 and the production of the ICD fragment that is translocated to the nucleus, is considered a key step during the apoptotic signaling pathway triggered by this receptor although the exact physiological effects of p75ICD release depends on the neuronal subtype [18, 40]. Recently, experimental evidence indicates that p75 signaling, including cleavage and release of the ICD, is indeed strongly related to $\mathrm{AD}$, highlighting the central role of p75 during AD pathogenesis [59]. Thus, several mechanisms have been proposed involving p75 signaling in $\mathrm{AD}$. For instance, it was shown that $\mathrm{A} \beta$ is able to bind to p75 and trigger activation of the receptor inducing the cleavage of the ICD [60]. Moreover, amyloidogenesis is aggravated by the interaction of beta-site amyloid precursor protein cleaving enzyme-1 (BACE1) with p75 upon the binding of $A \beta$ to $p 75$ [21]. In contrast, it was shown that the p75ECD has a neuroprotective effect against $A \beta$ toxicity [61] and that transmembrane domain of p75, has trophic effects by inducing phosphorylation of $\operatorname{TrKB}$ [62]. Our results emphasize the important contribution of p75 signaling, including p75ICD internalization and apoptosis, in $\mathrm{AD}$ pathogenesis upon AGE-modified proBDNF stimulation. More importantly, our data provide a novel pro-apoptotic mechanism in $\mathrm{AD}$ and involving p75 in which higher levels of cleavage due to mutations in PS1 make these neurons more sensitive to the adverse effects of AGE-modified proBDNF. Moreover, we suggest that this is a general mechanism in $\mathrm{AD}$, also for the non-familiar, spontaneous form of the disease, given the fact that $A \beta$ is able to trigger $p 75$ cleavage and p75ICD release and could therefore prime p75 signaling for AGE modified proBDNF independenty of PS1 mutations.

\section{Additional file}

Additional file 1: Figure S1. NSCs in culture express P75NTR, sortilin, DCX and SV2. Figure S2. NSCs in culture expressing DCX and SV2, are positives for TrkB. Figure S3. Characterization of Adult NSCs in culture. Figure S4. IDC p75NTR cellular distribution in NSC. (PDF $1196 \mathrm{~kb}$ )

\footnotetext{
Abbreviations

AD: Alzheimer disease; AGEs: ROS derived advanced glycation end products; ALEs: Advanced lipooxidation end products; BACE1: a-protease; BDNF: Brain-derived neurotrophic factor; BSA: Bovine Serum Albumin; CEL: NE-(carboxyethyl)-lysine; CML: Carboxymethyl)-lysine; CSF: Cerebrospinal fluid; CSFID: Immunodepleted CSF; DIV: Day in vitro; DMEM: Dulbecco's modified Eagle's medium; EGF: Epidermal Growth Factor; ELISA: Enzyme-immuno assay; FGF: Fibroblast Growth Factor; GO: Glyoxal; HNEL: 4-hydroxy-2-nonenalIysine; ICD: Intracellular domain; MBSA: MGO-modified BSA; MDAL: NE(malondialdehyde)-lysine; MGO: methylglyoxal; MMSE: Minimental state
}

examination; MproBDNF: MGO-modified proBDNF; ND: Neurodegeneration; NSCs: Hippocampal Neural Stem Cells; OS: Oxidative stress; P/S: Penicylin/ Streptomycin; PBS: Phosphate buffered saline; PBS-T: PBS with 0, 1\% Triton X-100; PFA: Paraformaldehyde; pH 8.0: 133 mM NaCl, 0.2\% Tween 20; proBDNF: Precursor form of BDNF; PS1: Presenilin 1; ROS: Radical Oxygen Species; SD: Standard deviation; TBS-T: $50 \mathrm{mM}$ Tris

\section{Acknowledgements}

We thank Ester Aso for caring transgenic mice, Jesus Moreno for helping in the management of human post-mortem samples, and Ricard Curia, and Andrea Rios for helping with different aspects of the experimental work. We thank Montse Ortega, Sonia Rius, Noel Perez and Marc Tarres and the animal house facility staff of the Unversity of Lleida for technical assistance.

\section{Funding}

This work was supported by "Fundació La Marató 2015" (C.E.). We thank, IRBLleida Biobank (B.0000682), PLATAFORMA BIOBANCOS PT13/0010/0014, HUB-ICO-IDIBELL Biobank for providing human tissue and UAI IRBLleida for management support.

\section{Availability of data and materials}

Data sharing is not applicable to this article as no datasets were generated or analysed during the current study.

\section{Authors' contributions}

All authors have participated in the work to take public responsibility for appropriate portions of the content; and agreed to be accountable for all aspects of the work in ensuring that questions related to the accuracy or integrity of any part of the work are appropriately investigated and resolved. $F C$ and $E C$ are responsible of the main part of the design of the work and of acquisition, analysis and interpretation of data. MP and RD contributed to the experimental work. PG is responsible of the obtention and analysis of the human CSF samples. Fl is responsible of the obtention and analysis of the human brain samples. RC, EJ and EC have been involved in drafting the manuscript and revising it critically. All authors read and approved the final manuscript.

\section{Ethics approval and consent to participate}

Brain samples were obtained from the Institute of Neuropathology, Bellvitge University Hospital. Brain tissue was obtained from the Institute of Neuropathology HUB-ICO-IDIBELL Biobank and the Hospital Clinic-IDIBAPS Biobank following the guidelines of Spanish legislation on this matter (Real Decreto de Biobancos 1716/ 2011) and approval of the local ethics committee.

For the obtention of CSF samples, all patients have signed informed consent and the study has been approved by the hospital's ethics committee. IRBLleida Biobank (B.0000682), PLATAFORMA BIOBANCOS PT13/0010/0014. Animal procedures were conducted according to ethical guidelines (European Communities Council Directive 86/609/EEC) and approved by the local ethics committee of the Universitat de Lleida.

\section{Consent for publication}

Not applicable.

\section{Competing interests}

The authors declare that they have no competing interests.

\section{Publisher's Note}

Springer Nature remains neutral with regard to jurisdictional claims in published maps and institutional affiliations.

\section{Author details}

'Molecular Developmental Neurobiology Group, IRBLleida-UDL Rovira Roure 82, 25198 Lleida, Spain. ${ }^{2}$ Departament de Patologia i Terapèutica Experimental, Universitat de Barcelona, Barcelona, Spain. ${ }^{3}$ Centro de Investigación Biomédica en Red de Enfermedades Neurodegenerativas (CIBERNED), Hospitalet de Llobregat, Barcelona, Spain. ${ }^{4}$ Unitat Trastorns Cognitius, IRBLleida-Hospital Universitari Santa Maria Lleida, Lleida, Spain. ${ }^{5}$ Centre de Recherches sur la Cognition Animale (CRCA), Centre de Biologie Intégrative (CBI), Université de Toulouse, CNRS, UPS, 31062 Toulouse, France. ${ }^{6}$ Serra Húnter fellow, Associate Professor, Generalitat de Catalunya, Barcelona, Spain. 
Received: 25 May 2018 Accepted: 25 October 2018

Published online: 14 November 2018

\section{References}

1. Pamplona R, Dalfó E, Ayala V, Bellmunt MJ, Prat J, Ferrer I, et al. Proteins in human brain cortex are modified by oxidation, glycoxidation, and lipoxidation. Effects of Alzheimer disease and identification of lipoxidation targets. J Biol Chem. 2005;280:21522-30.

2. Hennigan A, O'Callaghan RM, Kelly AM. Neurotrophins and their receptors: roles in plasticity, neurodegeneration and neuroprotection. Biochem. Soc. Trans. 2007;35:424-7.

3. Pezet $\mathrm{S}$, Malcangio M. Brain-derived neurotrophic factor as a drug target for CNS disorders. Expert Opin Ther Targets. 2004:8:391-9.

4. Barbacid M. Neurotrophic factors and their receptors. Curr Opin Cell Biol. 1995:7:148-55.

5. Lewin GR, Barde YA. Physiology of the neurotrophins. Annu Rev Neurosci. 1996;19:289-317.

6. Lee R, Kermani P, Teng KK, Hempstead BL. Regulation of cell survival by secreted proneurotrophins. Science. 2001;294:1945-8.

7. Beattie MS, Harrington AW, Lee R, Kim JY, Boyce SL, Longo FM, et al. ProNGF induces p75-mediated death of oligodendrocytes following spinal cord injury. Neuron. 2002;36:375-86

8. Nykjaer A, Lee R, Teng KK, Jansen P, Madsen P, Nielsen MS, et al. Sortilin is essential for proNGF-induced neuronal cell death. Nature. 2004;427:843-8.

9. Teng HK, Teng KK, Lee R, Wright S, Tevar S, Almeida RD, et al. ProBDNF induces neuronal apoptosis via activation of a receptor complex of p75 and Sortilin. J Neurosci. 2005;25:5455-63.

10. Massa SM, Xie Y, Yang T, Harrington AW, Kim ML, Yoon SO, et al. Small, nonpeptide p75 ligands induce survival signaling and inhibit proNGFinduced death. J Neurosci. 2006;26:5288-300.

11. Koshimizu H, Hazama S, Hara T, Ogura A, Kojima M. Distinct signaling pathways of precursor BDNF and mature BDNF in cultured cerebellar granule neurons. Neurosci Lett. 2010;473:229-32.

12. Barker PA. p75NTR is positively promiscuous: novel partners and new insights. Neuron. 2004:42:529-33.

13. López-Sánchez N, Rodríguez JR, Frade JM. Mitochondrial c-Jun NH2-terminal kinase prevents the accumulation of reactive oxygen species and reduces necrotic damage in neural tumor cells that lack trophic support. Mol Cancer Res. 2007;5(1):47-60.

14. Volosin M, Trotter C, Cragnolini A, Kenchappa RS, Light M, Hempstead BL, Carter BD, Friedman WJ. Induction of proneurotrophins and activation of p75-mediated apoptosis via Neurotrophin receptor interacting factor (NRIF) in hippocampal neurons after seizures. J Neurosci. 2008;28(39):9870-9.

15. Frade JM. Nuclear translocation of the $\mathrm{p} 75$ neurotrophin receptor cytoplasmic domain in response to neurotrophin binding. J Neurosci. 2005; 25(6):1407-11.

16. Podlesniy P, Kichev A, Pedraza C, Saurat J, Encinas M, Perez B, et al. ProNGF from Alzheimer's disease and normal human brain displays distinctive abilities to induce processing and nuclear translocation of intracellular domain of p75 and apoptosis. Am J Pathol. 2006:169(1):119-31.

17. Kenchappa RS, Tep C, Korade Z, Urra S, Bronfman FC, Yoon SO, et al. p75 neurotrophin receptor-mediated apoptosis in sympathetic neurons involves a biphasic activation of JNK and up-regulation of tumor necrosis factoralpha-converting enzyme/ADAM17. J Biol Chem. 2010;285(26):20358-68.

18. Parkhurst CN, Zampieri N, Chao MV. Nuclear localization of the p75 neurotrophin receptor intracellular domain. J Biol Chem. 2010;285(8):5361-8.

19. Kenchappa RS, Zampieri N, Chao MV, Barker PA, Teng HK, Hempstead BL, et al. Ligand-dependent cleavage ofthe P75 neurotrophin receptor is necessary for NRIF nuclear translocation and apoptosis in sympathetic neurons. Neuron. 2006;50:219-32

20. Chakravarthy B, Ménard M, Ito S, Gaudet C, Dal Prà I, Armato U, Whitfield J. Hippocampal membrane-associated p75NTR levels are increased in Alzheimer's disease. J Alzheimers Dis. 2012:30(3):675-84.

21. Saadipour K, Mañucat-Tan NB, Lim Y, Keating DJ, Smith KS, Zhong JH, Liao H, Bobrovskaya L, Wang YJ, Chao MV, Zhou XF. p75 neurotrophin receptor interacts with and promotes BACE1 localization in endosomes aggravating amyloidogenesis. J Neurochem. 2018;144(3):302-17.

22. Allen SJ, Wilcock GK, Dawbarn D. Profound and selective loss of catalytic TrkB immunoreactivity in Alzheimer's disease. Biochem Biophys Res Commun. 1999;264(3):648-51.
23. Ferrer I, Marin C, Rey MJ, Ribalta T, Goutan E, Blanco R, et al. BDNF and fulllength and truncated TrkB expression in Alzheimer disease. Implications in therapeutic strategies. J Neuropathol Exp Neurol. 1999;58(7):729-39.

24. Forlenza OV, Diniz BS, Teixeira AL, Radanovic $M$, Talib $L L$, Rocha NP, et al. Lower cerebrospinal fluid concentration of brain-derived neurotrophic factor predicts progression from mild cognitive impairment to Alzheimer's disease. NeuroMolecular Med. 2015;17:326-32.

25. Holsinger RM, Schnarr J, Henry P, Castelo VT, Fahnestock M. Quantitation of BDNF mRNA in human parietal cortex by competitive reverse transcriptionpolymerase chain reaction: decreased levels in Alzheimer's disease. Brain Res Mol Brain Res. 2000;76:347-54.

26. Hock C, Heese K, Hulette C, Rosenberg C, Otten U. Region-specific neurotrophin imbalances in Alzheimer disease: decreased levels of brainderived neurotrophic factor and increased levels of nerve growth factor in hippocampus and cortical areas. Arch Neurol. 2000;57:846-51.

27. Pedraza CE, Podlesniy P, Vidal N, Arévalo JC, Lee R, Hempstead B, et al. ProNGF isolated from the human brain affected by Alzheimer's disease induces neuronal apoptosis mediated by p75. Am J Pathol. 2005;166(2):533-43.

28. Kichev A, Ilieva EV, Piñol-Ripoll G, Podlesniy P, Ferrer I, Portero-Otín M, et al. Cell death and learning impairment in mice caused by in vitro modified proNGF can be related to its increased oxidative modifications in Alzheimer disease. Am J Pathol. 2009;175:2574-85.

29. Braak H, Alafuzoff I, Arzberger T, Kretzschmar H, Del Tredici K. Staging of Alzheimer disease-associated neurofibrillary pathology using paraffin sections and immunocytochemistry. Acta Neuropathol. 2006;112:389-404.

30. Braak H, Braak E. Demonstration of amyloid deposits and neurofibrillary changes in whole brain sections. Brain Pathol. 1991;1:213-6.

31. Borchelt DR, Thinakaran G, Eckman CB, Lee MK, Davenport F, Ratovitsky T, et al. Familial Alzheimer's disease-linked presenilin 1 variants elevate Abeta142/1-40 ratio in vitro and in vivo. Neuron. 1996;17:1005-13.

32. Aso E, Lomoio S, López-González I, Joda L, Carmona M, Fernández-Yagüe N, Moreno J, Juvés S, Pujol A, Pamplona R, Portero-Otin M, Martín V, Díaz M, Ferrer I. Amyloid generation and dysfunctional immunoproteasome activation with disease progression in animal model of familial Alzheimer's disease. Brain Pathol. 2012;22(5):636-53.

33. Carpenter AE, Jones TR, Lamprecht MR, Clarke $\mathrm{C}$, Kang $\amalg$, Friman $\mathrm{O}$, et al. CellProfiler: image analysis software for identifying and quantifying cell phenotypes. Genome Biol. 2006;7:R100.

34. Espinet C, Gonzalo H, Fleitas C, Menal MJ, Egea J. Oxidative stress and neurodegenerative diseases: a neurotrophic approach. Curr Drug Targets. 2015;16(1):20-30.

35. Uchida K, Shiraishi M, Naito Y, Torii Y, Nakamura Y, Osawa T. Activation of stress signaling pathways by the end product of lipid peroxidation. 4-hydroxy-2nonenal is a potential inducer of intracellular peroxide production. J.Biol Chem. 1999:274:2234-42

36. Stitt AW, Frizzell N, Thorpe SR. Advanced glycation and advanced lipoxidation: possible role in initiation and progression of diabetic retinopathy. Curr Pharm Des. 2004;10(27):3349-60.

37. Lanoiselée HM, Nicolas G, Wallon D, Rovelet-Lecrux A, Lacour M, Rousseau S, et al. APP, PSEN1, and PSEN2 mutations in early-onset Alzheimer disease: A genetic screening study of familial and sporadic cases. PLoS Med. 2017:14(3):e1002270.

38. Pigino G, Pelsman A, Mori H, Busciglio J. Presenilin-1 mutations reduce cytoskeletal association, deregulate neurite growth, and potentiate neuronal dystrophy and tau phosphorylation. J Neurosci. 2001;21(3):834-42.

39. Lessard CB, Wagner SL, Koo EH. And four equals one: presenilin takes the gamma-secretase role by itself. Proc Natl Acad Sci U S A. 2010;107(50):21236-7.

40. Vicario A, Kisiswa L, Tann JY, Kelly CE, Ibanez CF. Neuron-type specific signaling by the p75NTR death receptor is regulated by differential proteoloytic cleavage. J Cell Sci. 2015;128:1507-17.

41. Webster SJ, Bachstetter AD, Nelson PT, Schmitt FA, Van Eldik LJ. Using mice to model Alzheimer's dementia: an overview of the clinical disease and the preclinical behavioral changes in 10 mouse models. Front Genet. 2014;5:88.

42. Fahnestock M, Michalski B, Xu B, Coughlin MD. The precursor pro-nerve growth factor is the predominant form of nerve growth factor in brain and is increased in Alzheimer's disease. Mol Cell Neurosci. 2001;18(2):210-20.

43. Pang PT, Lu B. Regulation of late-phase LTP and long-term memory in normal and aging hippocampus: role of secreted proteins tPA and BDNF. Ageing Res Rev. 2004;3(4):407-30.

44. Matsumoto T, Rauskolb S, Polack M, Klose J, Kolbeck R, Korte M, Barde YA. Biosynthesis and processing of endogenous BDNF: CNS neurons store and secrete BDNF, not proBDNF. Nat Neurosci. 2008;11(2):131-3. 
45. Yang J, Siao C-J, Nagappan G, Marinic T, Jing D, McGrath K, et al. Neuronal release of proBDNF. Nat Neurosci. 2009;12:113-5.

46. Chen ZY, Jing D, Bath KG, leraci A, Khan T, Siao CJ, Herrera DG, Toth M, Yang C, BS ME, Hempstead BL, Lee FS. Genetic variant BDNF (Val66Met) polymorphism alters anxiety-related behavior. Science. 2006;314(5796):140-3.

47. Zhang Q, Ames JM, Smith RD, Baynes JW, Metz TO. A perspective on the Maillard reaction and the analysis of protein glycation by mass spectrometry: probing the pathogenesis of chronic disease. J Proteome Res. 2009;8:754-69.

48. Smerjac SM, Bizzozero OA. Cytoskeletal protein carbonylation and degradation in experimental autoimmune encephalomyelitis. J Neurochem. 2008;105:763-72

49. Harris RA, Amor S. Sweet and sour--oxidative and carbonyl stress in neurological disorders. CNS Neurol Disord Drug Targets. 2011;10:82-107.

50. Ullrich A, Gray A, Berman C, Dull TJ. Human beta-nerve growth factor gene sequence highly homologous to that of mouse. Nature. 1983;303:821-5.

51. Voineskos AN, Lerch JP, Felsky D, Shaikh S, Rajji TK, Miranda D, et al. The brain-derived neurotrophic factor Val66Met polymorphism and prediction of neural risk for Alzheimer disease. Arch Gen Psychiatry. 2011;68:198-206.

52. Anastasia A, Deinhardt K, Chao MV, Will NE, Irmady K, Lee FS, et al. Val66Met polymorphism of BDNF alters prodomain structure to induce neuronal growth cone retraction. Nat Commun. 2013;4:2490

53. Egan MF, Kojima M, Callicott JH, Goldberg TE, Kolachana BS, Bertolino A, et al. The BDNF val66met polymorphism affects activity-dependent secretion of BDNF and human memory and hippocampal function. Cell. 2003;1 12:257-69.

54. Chen Z-Y. Variant brain-derived neurotrophic factor (BDNF) (Met66) alters the intracellular trafficking and activity-dependent secretion of wild-type BDNF in neurosecretory cells and cortical neurons. J Neurosci. 2004;24:4401-11.

55. Yu H, Wang D-D, Wang Y, Liu T, Lee FS, Chen Z-Y. Variant brain-derived neurotrophic factor Val66Met polymorphism alters vulnerability to stress and response to antidepressants. J Neurosci. 2012;32:4092-101.

56. Ji H, Da D, Wang Y, Jiang D, Zhou X, Lin P, et al. Association of BDNF and BCHE with Alzheimer's disease: Meta-analysis based on 56 genetic case-control studies of 12,563 cases and 12,622 controls. Exp. Ther. Med. 2015;9:1831-40.

57. Al-Shawi R, Hafner A, Chun S, Raza S, Crutcher K, Thrasivoulou C, Simons P, Cowen T. ProNGF, sortilin, and age-related neurodegeneration. Ann N Y Acad Sci. 2007;1119:208-15.

58. Saadipour K, Yang M, Lim Y, Georgiou K, Sun Y, Keating D, et al. Amyloid beta $1-42^{-4}\left(A \beta_{42}\right)$ up-regulates the expression of Sortilin via the p75(NTR)/ RhoA signaling pathway. J Neurochem. 2013:127(2):152-62.

59. Chao MV. Cleavage of p75 neurotrophin receptor is linked to Alzheimer's disease. Mol Psychiatry. 2016;21(3):300-1.

60. Sotthibundhu A, Sykes AM, Fox B, Underwood CK, Thangnipon W, Coulson EJ. Beta-amyloid(1-42) induces neuronal death through the p75 neurotrophin. Receptor J Neurosci. 2008;28(15):3941-6.

61. Yao XQ, Jiao SS, Saadipour K, Zeng F, Wang QH, Shen LL, et al. p75NTR ectodomain is a physiological neuroprotective molecule against amyloid-beta toxicity in the brain of Alzheimer's disease. Mol Psychiatry. 2015;20:1301-10.

62. Saadipour K, MacLean M, Pirkle S, Ali S, Lopez-Redondo ML, Stokes DL, Chao MV. The transmembrane domain of the p75 neurotrophin receptor stimulates phosphorylation of the TrkB tyrosine kinase receptor. J Biol Chem. 2017;292(40):16594-604.

Ready to submit your research? Choose BMC and benefit from:

- fast, convenient online submission

- thorough peer review by experienced researchers in your field

- rapid publication on acceptance

- support for research data, including large and complex data types

- gold Open Access which fosters wider collaboration and increased citations

- maximum visibility for your research: over $100 \mathrm{M}$ website views per year

At BMC, research is always in progress.

Learn more biomedcentral.com/submissions 